1 Ministério da Saúde, Secretaria Executiva - Brasília (DF), Brasil. Prefeitura de São Paulo, Secretaria Municipal da Saúde - São Paulo (SP),

Brasil.

celia.aly@gmail.com

2 Ministério da Saúde, Secretaria Executiva - Brasília (DF), Brasil. Prefeitura de Belo Horizonte, Secretaria Municipal da Saúde - Belo Horizonte (MG), Brasil.

afonso.reis@saude.gov.br

${ }^{3}$ Ministério da Previdência Social, Instituto Nacional do Seguro Social (INSS) - Brasília (DF). Prefeitura de São Paulo, Secretaria Municipal da Saúde - São Paulo (SP), Brasil.

ser.carneiro@gmail.com

${ }^{4}$ Ministério da Saúde, Secretaria Executiva Brasília (DF). Universidade do Sul de Santa Catarina (Unisul) - Florianópolis (SC), Brasil.

lucischeffer@gmail.com

\section{o Sistema Único de Saúde em série histórica de indicadores: uma perspectiva nacional para ação}

\author{
The Unified Health System in a historical series of indicators: a \\ national perspective for action
}

Célia Maria Castex Aly², Afonso Teixeira dos Reis², Sérgio Antonio Martins Carneiro ${ }^{\mathbf{3}}$, Luci

Fabiane Scheffer Moraes ${ }^{4}$

RESUMO Este artigo analisa a série histórica de um conjunto de indicadores, de 2002 a 2014, relacionados ao Sistema Único de Saúde do Brasil, embasado na metodologia da Proposta de Avaliação de Desempenho do Sistema de Saúde. Os resultados mostram que houve uma sensível melhoria nos indicadores de dimensão socioeconômica e nos da dimensão condições de saúde. A melhoria dos indicadores de condições de saúde pode estar relacionada ao incremento de suporte financeiro; ao incremento de recursos humanos; ao aumento do acesso às consultas médicas e aos serviços de alta complexidade; e a uma maior disponibilização de horas de profissionais de saúde para a população residente.

PALAVRAS-CHAVE Avaliação em saúde. Sistema Único de Saúde. Indicadores.

ABSTRACT This article examines the historical series of a set of indicators, from 2002 to 2014, related to the Unified Health System of Brazil, based on the methodology of the Proposal for Evaluation of Performance of the Health System. The results show that there has been a significant improvement in the socioeconomic dimension indicators and in the health conditions dimension. The improvement of the indicators of health conditions may be related to the increase of financial support; increase of human resources; increase of access to medical appointments and services of high complexity; and a greater availability of hours of health professional to the resident population.

KEYWORDS Health evaluation. Unified Health System. Indicators. 


\section{Introdução}

Monitorar e avaliar permanentemente as políticas públicas responde a uma antiga demanda de setores da sociedade por uma gestão pública de excelência e planejada, por um modelo de gestão dos recursos públicos (SANTOS, 2015). Esse movimento, que tem se fortalecido desde meados da década de 1990 do século $\mathrm{XX}$, provocou a estruturação de sistemas de monitoramento que, mesmo de forma limitada, são capazes de capturar desempenhos, mostrar tendências e apoiar os gestores no planejamento institucional e na prestação de contas à sociedade (JANNUZZI, 2012).

A criação da Rede Interagencial de Informações para a Saúde (Ripsa), em 1996, foi fruto do esforço de múltiplas parcerias institucionais. A Ripsa se caracteriza por ser uma agência de caráter participativo das instituições associadas e, desde 1997, sistematiza periodicamente um conjunto de indicadores que mobiliza e tem provocado o monitoramento e a avaliação de pactos institucionais nas três esferas de governo no Sistema Único de Saúde (SUS) (RIPSA, 2008). A institucionalização dos pactos foi outra expressão desse movimento, a exemplo do que ocorreu, entre 2001 e 2006, com o Pacto da Atenção Básica (BRASIL, 2003), e com o Pacto pela Saúde ocorrido, entre 2006 e 2011 (BRASIL, 2006), além da criação do Índice de Desempenho do SUS (IDSUS) (IDSUS, 2012) e do Pacto do Rol de Diretrizes, Objetivos, Metas e Indicadores 2013-2015, desde 2013 (BRASIL, 2015).

O Ministério da Saúde (MS), no sentido de apoiar as gestões estaduais e municipais, desenvolveu e tem disponibilizado aferições sistemáticas e coordenadas de indicadores de saúde e de desempenho do sistema brasileiro de saúde, seja para subsidiar análises estratégicas e operacionais, seja para monitorar e avaliar a concretização dos objetivos do SUS, no acompanhamento crítico e qualificado de metas, na prestação de contas e na melhoria da qualidade de serviços oferecidos à população brasileira.
A Secretaria de Gestão Estratégica e Participativa (Segep), criada em 2003, está fundamentada na prática do monitoramento e da avaliação das esferas de gestão estadual e municipal e no controle social participativo na gestão federal. Indo nessa direção, em 2011, é criado o Departamento de Monitoramento e Avaliação do SUS (Demas/SE/MS), com a finalidade de desenvolver e aperfeiçoar mecanismos, sistemas e modelos analíticos de Monitoramento e Avaliação (M\&A).

Em 2012, o Demas/SE/MS criou o IDSUS, resultante de um processo participativo, com consulta pública e participação ativa de gestores, da comunidade científica, de trabalhadores e usuários do SUS. O IDSUS reúne um conjunto de indicadores simples e compostos que buscam fazer uma aferição contextualizada do desempenho do SUS quanto à efetivação de seus princípios norteadores e diretrizes organizativas para a atenção à saúde.

Em uma escala de 0 a 10, o Brasil apresentou um IDSUS de 5,47. A região Sul teve um IDSUS de 6,12; seguida do Sudeste, com 5,56; Nordeste, com 5,28; Centro-Oeste, com 5,26 ; e Norte, com 4,67. Os estados que apresentaram os índices mais altos foram Santa Catarina, com 6,29; Paraná, com 6,23; e Rio Grande do Sul, com 5,90. Os menores índices foram Pará, 4,17; Rondônia, com 4,49; e Rio de Janeiro, com 4,58 (MEDICI, 2012).

O IDSUS foi uma importante iniciativa de apoio à gestão, por possibilitar monitoramento e avaliação na implantação e no desenvolvimento de políticas, ações e projetos de saúde ao longo do tempo, porém, a divulgação do índice expôs fragilidades de gestão e provocou controvérsias burocráticas, técnicas e políticas, que geraram a interrupção da mensuração e do aprimoramento do índice.

Concomitantemente à reavaliação do IDSUS, o Demas/SE/MS optou por mostrar o desempenho do SUS através da descrição de indicadores em série temporal, considerando que essa forma de apresentação auxiliaria a avaliação da implantação e do desenvolvimento de políticas, ações e projetos ao longo do tempo. 
A descrição articulada de um conjunto de indicadores propiciou a criação do 'O SUS em vista', no qual os indicadores relacionados ao setor saúde são classificados em 4 dimensões: socioeconômicas, de saúde, de financiamento e de serviços de saúde do SUS, com dados referentes, no mínimo, ao ano de 2002, até 2014 devido ao acesso aos dados atualizados. Os indicadores foram apresentados de forma descritiva através de frequências e proporções em tabelas, gráficos ou mapas.

Este artigo tem como objetivo analisar a série histórica de um conjunto de indicadores, no período de 2002 a 2014, relacionados ao desempenho do SUS, tendo como referência o 'O SUS em vista'.

\section{Estratégia metodológica}

Os indicadores deste estudo foram selecionados a partir dos resultados produzidos pelo Demas/ SE/MS (BRASIL, 2016), intitulado 'O SUS em vista', e foram reagrupados segundo a Matriz de Dimensões da Avaliação de Desempenho do Sistema de Saúde (FIOCRUZ, 2003; 2011).

Os critérios de escolha dos indicadores incluídos nesta análise foram: a disponibilidade e a completitude dos dados entre os anos de 2002 a 2014; o poder de síntese do indicador; não apresentar similaridade com outro indicador; e ter relevância para a gestão do SUS. Nesse sentido, o presente estudo relaciona um número menor de indicadores e de subdimensões do que o modelo do instrumento de Metodologia de Avaliação do Desempenho do Sistema de Saúde (Proadess) propõe.

As fontes de dados dos indicadores são do MS e do Instituto Brasileiro de Geografia e Estatística (IBGE). São elas: Cadastro Nacional de Estabelecimentos de Saúde (CNES); Fundo Nacional de Saúde (FNS); Sistema de Informações sobre Mortalidade (SIM); Sistema de Informações sobre Nascidos Vivos (Sinasc); Sistema de Informação Ambulatorial (SIA/SUS); Sistema de Informações Hospitalares do
SUS (SIH/SUS); Sistema de Informações sobre Orçamentos Públicos em Saúde (Siops/MS); Pesquisa Nacional por Amostra de Domicílio - Suplemento Saúde, de 2003 e 2008 (PNAD-Saúde); Pesquisa Nacional por Amostra de Domicílio anual (PNAD); Pesquisa Nacional de Saúde (PNS), de 2013; Censo Demográfico de 2012; e estimativas populacionais. Ressalta-se que em 2010 não houve a PNAD devido à realização do censo.

Desse modo, foi possível agrupar os indicadores nas seguintes dimensões da metodologia do Proadess:

\section{Dimensão socioeconômica da saúde}

Foram escolhidos 3 indicadores, que são explicativos da tendência da saúde de um grupo populacional. Os três indicadores apreciados são legitimados e compõem compromissos internacionais do Estado brasileiro (RIPSA, 2008), inclusive, seu monitoramento extrapola a competência exclusiva do MS, compondo a agenda interministerial (BRASIL, 2011). São eles: 1) Produto Interno Bruto (PIB) per capita; 2) Proporção de Pessoas de 10 anos ou mais de idade, sem ou com menos de um ano de instrução; e 3) Proporção da população com renda até meio salário mínimo.

\section{Indicadores de condições de saúde da população}

Na dimensão condição de saúde, foram escolhidos dois indicadores que se destacam pela consistência e pelo significado específico, já amplamente apresentados pela literatura pertinente, além de sinalizarem a interface das ações de saúde com outras ações, programas e políticas sociais em curso (RIPSA, 2008). São eles: 1) Taxa de mortalidade infantil e 2) Esperança de vida ao nascer.

\section{Dimensão de financiamento do siste- ma de saúde}

Os indicadores selecionados referem-se à 
composição das fontes de financiamento e à distribuição da despesa pública em saúde. Foram selecionados três, sendo eles: 1) Despesas do SUS em ações e serviços públicos de saúde financiados por recursos próprios nas três esferas de governo; 2) Transferências SUS, fundo a fundo, da União a municípios, estados e DF; e 3) Médias per capita das despesas em saúde das três esferas de governo.

\section{Dimensão de recursos humanos do sistema de saúde}

Os indicadores selecionados referem-se às pessoas inseridas no sistema de saúde que exercem uma ocupação e possuem uma formação profissional. Foram selecionados quatro: 1) média mensal do número de Equipes de Saúde da Família (EqSF); 2) razão entre horas semanais de médicos e população residente, na atenção básica; 3) razão de horas semanais dos enfermeiros e da população residente, na atenção básica; e 4) razão de horas semanais de outros profissionais de saúde de nível superior, na atenção básica.

\section{Dimensão de acesso aos serviços de saúde}

Os indicadores selecionados expressam a capacidade das pessoas de obter os serviços necessários no lugar e no momento certo. Foram selecionados cinco indicadores. São eles: 1) Razão de internações clínicas de média complexidade, sensíveis à atenção básica, de residentes e população de mesma residência; 2) Razão de internações clínicas de média complexidade, excluídas as sensíveis à atenção básica, de residentes e população de mesma residência; 3) Razão de internações clínico-cirúrgicas de alta complexidade de residentes e população de mesma residência; 4) Proporção de pessoas que consultaram médico nos últimos 12 meses; e 5) Proporção da população que referiu internação hospitalar nos últimos 12 meses.

O quadro 1 apresenta os indicadores selecionados conforme objetivos, método de cálculo, unidade de medida, fonte e período de análise.

Quadro 1. Conjunto de indicadores relacionados ao desempenho do SUS selecionados devido a sua completitude e relevância para a análise das dimensões de saúde no Brasil, no período de 2002 a 2014.

\begin{tabular}{|c|c|c|c|c|}
\hline Indicador & Objetivo & Método de Cálculo e unidade de medida & Fonte & $\begin{array}{l}\text { Período de } \\
\text { análise }\end{array}$ \\
\hline \multicolumn{5}{|c|}{ DIMENSÃO SOCIOECONÔMICA DA SAÚDE } \\
\hline $\begin{array}{l}\text { Produto Interno Bruto } \\
\text { per capita }\end{array}$ & $\begin{array}{l}\text { Indicar o desenvolvimento econômico } \\
\text { do País }\end{array}$ & $\begin{array}{l}\text { Valor do produto interno bruto / População residente } \\
\text { Unidade de medida: mil reais* por pessoa }\end{array}$ & IBGE & $2002-2014$ \\
\hline $\begin{array}{l}\text { Proporção de pessoas, } \\
\text { com } 10 \text { ou mais anos } \\
\text { de idade, sem e com } \\
\text { menos de } 1 \text { ano de } \\
\text { instrução }\end{array}$ & $\begin{array}{l}\text { Indicar o nível de analfabetismo nas } \\
\text { pessoas com } 10 \text { anos ou mais. }\end{array}$ & $\begin{array}{l}\text { (№ de pessoas analfabetas }+ \text { no de pessoas com } \\
\text { menos de } 1 \text { ano de instrução / População residente no } \\
\text { território e ano avaliado) X } 100 \\
\text { Unidade de medida: porcentagem }\end{array}$ & PNAD & $2002-2014$ \\
\hline $\begin{array}{l}\text { Proporção da população } \\
\text { com renda até meio } \\
\text { salário mínimo }\end{array}$ & $\begin{array}{l}\text { Indicar a proporção da população em } \\
\text { situação de pobreza }\end{array}$ & $\begin{array}{l}\text { (População residente com renda domiciliar mensal } \\
\text { de até meio salário mínimo / no da população total } \\
\text { residente no mesmo território e ano avaliado) X } 100 \\
\text { Unidade de medida: porcentagem }\end{array}$ & PNAD & $2002-2014$ \\
\hline
\end{tabular}


Quadro 1. (cont.)

\section{DIMENSÃO DE CONDIC̣ÕES DE SAÚDE DA POPULAC̣ÃO}

Taxa de mortalidade infantil

Esperança de vida ao nascer
Estimar o risco de morte dos nascidos vivos durante o seu primeiro ano de vida

Indicar as condições de desenvolvimento socioeconômico.

Indicar o acesso e a qualidade dos recursos disponíveis para atenção à saúde materna e da população infantil

Avaliar os níveis de vida e de saúde da população
( $N$ o de óbitos com menos de 1 ano de idade)/

( $n$ ํ de nascidos vivos de mesma residência do ano avaliado) $\times 1000$

Unidade de medida: no de óbitos a cada mil habitan-

tes

A partir de tábuas de vida elaboradas para cada área geográfica, toma-se o número correspondente a uma geração inicial de nascimentos (IO) e determina-se o tempo cumulativo vivido por essa mesma geração (TO) até a idade limite. A esperança de vida ao nascer é o quociente da divisão de TO por 10

Unidade de medida: anos

SIM e

$2002-2013$

Sinasc

\section{DIMENSÃO DE FINANCIAMENTO DO SISTEMA DE SAÚDE}

Despesas do SUS em ações e serviços públicos de saúde financiados por recursos próprios nas três esferas de governo

Transferências SUS, fundo a fundo, da União a municípios, estados e DF

Médias per capita das despesas em saúde das três esferas de governo
Indicar o total de despesas do SUS nas três esferas de governo em ações e serviços públicos de saúde

Indicar o total de recursos financeiros da União transferidos aos municípios, estados e DF

Indicar a despesa média por habitante em ações e serviços públicos de saúde
Soma do total de despesas de cada ente federativo, com recursos próprios em ações e serviços públicos em saúde, atualizado pelo IPCA

Unidade de medida: bilhões de $\mathrm{R}$ \$*

Soma do total de recursos financeiros transferidos

para os estados, Distrito Federal e municípios de dotações consignadas nos Orçamentos Fiscais e da Seguridade Social da União. Unidade de medida: bilhões de $R \$ \star$

Despesas em saúde das três esferas do governo / População residente Unidade de medida: mil reais ${ }^{\star}$ por pessoa

Siops

2002-2014 IBGE
Média mensal do núme-

Média mensal do núme ro de Equipes de Saúde da Família

Razão entre horas semanais de médicos, na atenção básica e população residente

Razão entre horas semanais de enfermeiros na atenção básica e população residente Razão entre horas semanais de outros profissionais de saúde de nível superior, na atenção básica e população residente

\section{DIMENSÃO DE RECURSOS HUMANOS DO SISTEMA DE SAÚDE}

Indicar o número médio de equipes no País

Indicar a carga horária dos médicos realizada na atenção básica

Indicar a carga horária dos enfermeiros realizada na atenção básica

Indicar a carga horária dos outros profissionais de saúde de nível superior realizada na atencão básica
Soma do $n$ ํ de equipes registradas no SCNES / 12

Unidade de medida: no médio de equipes

(Soma anual das cargas horárias semanais de médicos na atenção básica / 12 X População residente) × 10000

Unidade de medida: hora média

(Soma anual das cargas horárias semanais de auxiliares e técnicos de enfermagem / 12 X População residente) $\times 10000$

Unidade de medida: hora média

(Soma anual das cargas horárias semanais de outros SCNES profissionais de saúde de nível superior / 12 X Popula- e IBGE cão residente) $\times 10000$

Unidade de medida: hora média

e IBGE

SCNES e IBGE

SCNES e IBGE

SCNES 2008-2014 2008-2014

2008-2014

洒

(1)

(10)


Quadro 1. (cont.)

\section{DIMENSÃO DE ACESSO DO SISTEMA DE SAÚDE}

\begin{tabular}{|c|c|c|c|c|}
\hline $\begin{array}{l}\text { Razão entre interna- } \\
\text { ções clínicas de média } \\
\text { complexidade, sensíveis } \\
\text { à atenção básica, de } \\
\text { residentes e população } \\
\text { de mesma residência }\end{array}$ & $\begin{array}{l}\text { Estimar a oferta de internações clínicas } \\
\text { de média complexidade, sensíveis à } \\
\text { atenção básica }\end{array}$ & $\begin{array}{l}\text { (№ de internações anuais de clínicas de média } \\
\text { complexidade, de residentes, devidas às condições } \\
\text { sensíveis à atenção básica / População residente no } \\
\text { território e ano avaliado) X } 100 \\
\text { Unidade de medida: no de internações a cada } 100 \\
\text { habitantes }\end{array}$ & $\begin{array}{l}\text { SIH/ } \\
\text { SUS e } \\
\text { IBGE }\end{array}$ & $2008-2014$ \\
\hline $\begin{array}{l}\text { Razão entre interna- } \\
\text { ções clínicas de média } \\
\text { complexidade, excluídas } \\
\text { as sensíveis à atenção } \\
\text { básica, de residentes e } \\
\text { população de mesma } \\
\text { residência }\end{array}$ & $\begin{array}{l}\text { Estimar a oferta de internações clínicas } \\
\text { de média complexidade, excluídas as } \\
\text { sensíveis à atenção básica }\end{array}$ & $\begin{array}{l}\text { (№ de internações anuais de clínicas de média com- } \\
\text { plexidade de residentes em um território, excluídas } \\
\text { aquelas sensíveis à atenção básica / População resi- } \\
\text { dente no mesmo território e ano avaliado) X } 100 \\
\text { Unidade de medida: no de internações a cada } 100 \\
\text { habitantes }\end{array}$ & $\begin{array}{l}\text { SIH/ } \\
\text { SUS e } \\
\text { IBGE }\end{array}$ & $2008-2014$ \\
\hline $\begin{array}{l}\text { Razão entre internações } \\
\text { clínico-cirúrgicas de alta } \\
\text { complexidade de resi- } \\
\text { dentes e população de } \\
\text { mesma residência }\end{array}$ & $\begin{array}{l}\text { Estimar a oferta de internações clínico- } \\
\text {-cirúrgicas de alta complexidade }\end{array}$ & $\begin{array}{l}\text { (№ de internações anuais de internações clínico- } \\
\text {-cirúrgicas de alta complexidade, de residentes / } \\
\text { População residente do mesmo território e ano ava- } \\
\text { liado) X } 1000 \\
\text { Unidade de medida: no de internações a cada } 100 \\
\text { habitantes }\end{array}$ & $\begin{array}{l}\mathrm{SIH/} \\
\text { SUS e } \\
\text { IBGE }\end{array}$ & $2008-2014$ \\
\hline $\begin{array}{l}\text { Proporção de pessoas } \\
\text { que consultaram médico } \\
\text { nos últimos } 12 \text { meses }\end{array}$ & $\begin{array}{l}\text { Estimar a oferta de consultas médicas } \\
\text { da população brasileira }\end{array}$ & $\begin{array}{l}\text { (№ de residentes com informação de ter consultado } \\
\text { médico pelo menos } 1 \text { vez, nos últimos } 12 \text { meses / } \\
\text { Total de residentes dos domicílios selecionados, no } \\
\text { mesmo território e ano, avaliados) X } 100 \\
\text { Unidade de medida: porcentagem }\end{array}$ & $\begin{array}{l}\text { PNAD- } \\
\text {-Saúde } \\
\text { e PNS }\end{array}$ & $\begin{array}{l}2003,2008 \\
\text { e } 2013\end{array}$ \\
\hline $\begin{array}{l}\text { Proporção da população } \\
\text { que refere internação } \\
\text { hospitalar nos últimos } \\
12 \text { meses }\end{array}$ & $\begin{array}{l}\text { Estimar a oferta de internação hospitalar } \\
\text { à população brasileira }\end{array}$ & $\begin{array}{l}\text { (Quantidade de residentes com informação de inter- } \\
\text { nação hospitalar, por } 24 \text { horas ou mais, nos últimos } 12 \\
\text { meses / Total de residentes dos domicílios seleciona- } \\
\text { dos, no mesmo território e ano, avaliados) } \\
\text { X } 100 \\
\text { Unidade de medida: porcentagem }\end{array}$ & $\begin{array}{l}\text { PNAD- } \\
\text {-Saúde } \\
\text { e PNS }\end{array}$ & $\begin{array}{l}2003,2008 \\
\text { e } 2013\end{array}$ \\
\hline
\end{tabular}

*Os valores em R\$ foram corrigidos pelo Índice Nacional de Preços ao Consumidor Amplo (IPCA), acumulado mensalmente, de janeiro de 2000 a dezembro de 2014.

\section{Resultados e discussão}

\author{
A figura 1 apresenta a série histórica e a evo- \\ lução dos indicadores socioeconômicos, de
}

condições de saúde, do financiamento do SUS e de acesso aos serviços de saúde, no período de 2002 a 2014. 
Figura 1. Série história e a evolução dos indicadores socioeconômicos, de condições de saúde, de acesso aos serviços de saúde e de financiamento do SUS, no período de 2002 a 2014
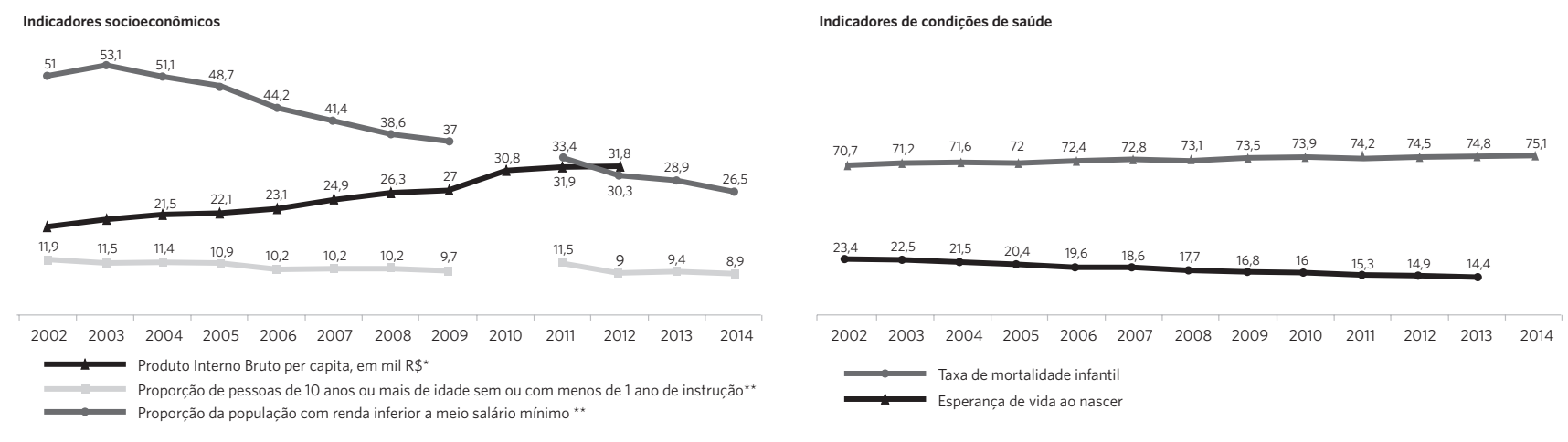

Indicadores de financiamento do SUS

Indicadores de acesso aos serviços de saúde
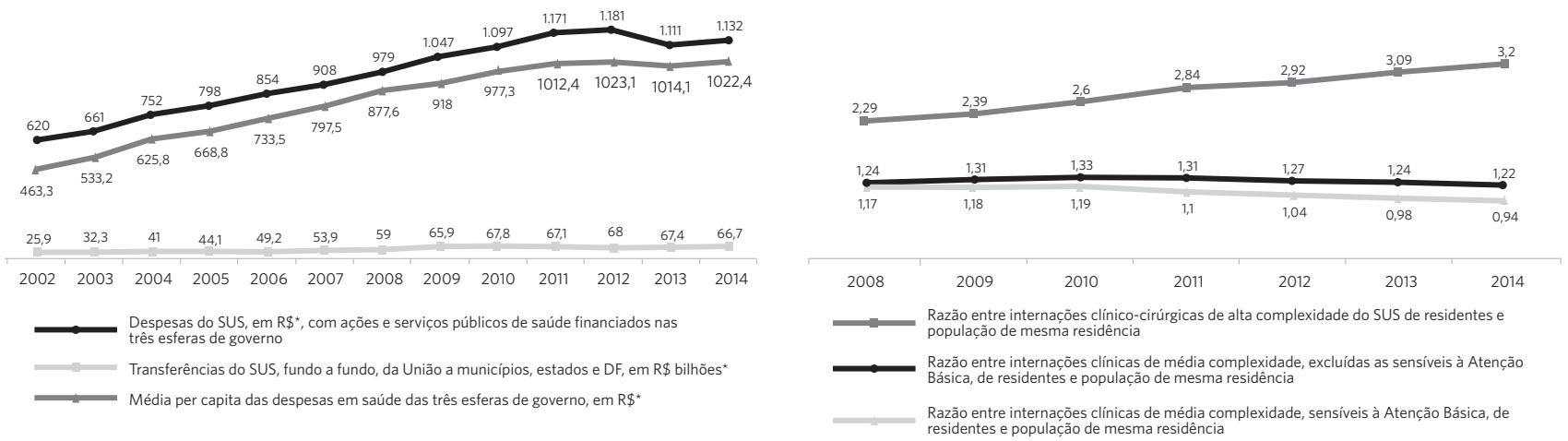

*Os valores em R\$ foram corrigidos pelo Índice Nacional de Preços ao Consumidor Amplo (IPCA), acumulado mensalmente, de janeiro de 2000 a dezembro de 2014.

${ }^{\star \star}$ A PNAD, em 2010, não foi realizada.

\section{Indicadores socioeconômicos}

Observa-se que o PIB per capita teve um aumento de $65,6 \%$ entre 2002 e 2012, passando de $\mathrm{R} \$ 19.200$, em 2002, para $\mathrm{R} \$ 31.800$, em 2012. O PIB per capita apresentou aumento ano após ano, entre 2002 e 2011, e uma queda leve de $0,3 \%$ entre 2011 e 2012. Segundo o Instituto de Pesquisa Econômica Aplicada (Ipea) (IPEA, 2012), em 2011, o Brasil obteve seu menor nível de desigualdade de renda desde os registros nacionais, iniciados em 1960, e destaca que, em 2003, registrou-se o fim de um ciclo de recessão iniciado em 1980. Esse quadro de maior produção e distribuição de renda é fruto de políticas de governo que visam a uma maior justiça social.
Houve a redução de $24,4 \%$ na proporção de pessoas analfabetas ao se compararem os dados de 2002 com os de 2014, que passaram de $11,9 \%$ para $8,9 \%$. Ressalta-se que esse indicador mede o percentual de pessoas com 10 anos de idade ou mais sem instrução e das pessoas com menos de um ano de instrução, sendo que, quanto menor o percentual, melhor o nível de instrução da população brasileira nessa faixa etária.

O Brasil, que possui a $5^{\text {a }}$ maior população do mundo, segundo o Comunicado $\mathrm{n}^{\mathrm{o}}$ 7 do Ipea (IPEA, 2010), ocupa a $8^{a}$ posição em números absolutos de analfabetos. Ao ser comparado com os países selecionados no referido comunicado, verifica-se que o Brasil encontra-se em uma posição intermediária. 
De um lado, o Paquistão, com a taxa de analfabetismo de $46,3 \%$, e do outro, o Chile, com $1,4 \%$. Embora o analfabetismo ainda se configure como um problema social, pode-se afirmar que a redução do analfabetismo indica uma melhoria do nível educacional brasileiro, resultante de um maior investimento governamental.

Houve a redução de 40,6\% na proporção de pessoas com renda inferior a meio salário mínimo, ao se comparar 2002 com 2014, que passou de $51 \%$ para $26,5 \%$. Essa redução verificada vai ao encontro de estudos que mostram que os índices de pobreza e extrema pobreza caíram rapidamente nos últimos 10 anos e que o perfil da pobreza no Brasil foi bastante e frequentemente alterado para melhor (CAMPELLO, 2013).

\section{Indicadores de condições de saúde}

Os indicadores de condição de saúde selecionados apresentam a evolução da taxa de mortalidade infantil e da esperança de vida ao nascer, no período de 2002 a 2014. Após análise desses indicadores, verificou-se uma redução na taxa de mortalidade infantil de $36,3 \%$ ao se comparar 2002 com 2013, passando de 23,4 para 14,4 mortes por mil habitantes. Segundo o 'Relatório Nacional de Acompanhamento dos Objetivos de Desenvolvimento do Milênio',

medidas de combate à pobreza e à desnutrição e as iniciativas de promoção da saúde da criança, como a oferta de vacinas gratuitas a todas as crianças, foram capazes de reduzir, de modo significativo, a mortalidade infantil. (IPEA, 2014, P. 10).

Com relação à esperança de vida ao nascer, verifica-se o aumento de 4,4 anos em 12 anos, passando de 70,7 anos, em 2002, para 75,1 anos, em 2014. A literatura científica mostra a estreita relação causal entre diminuição da mortalidade infantil e esperança de vida (DUARTE ET AL., 2002; GESSER, 2005).
O aumento da esperança de vida ao nascer indica melhoria das condições de vida e de saúde da população.

Cabe ressaltar que tanto a mortalidade infantil como a esperança de vida ao nascer são indicadores síntese de desenvolvimento socioeconômico por apresentarem consistência e capacidade de discriminação para afirmar melhoria das condições de vida.

\section{Indicadores de financiamento do SUS}

Os investimentos financeiros com o SUS aumentaram em 90,5\%, entre 2002 e 2014, com ações e serviços públicos de saúde financiados por recursos próprios nas três esferas de governo, passando de R $\$ 620 \mathrm{mil}$ para $\mathrm{R} \$ 1.132$ bilhões de reais, configurando um aumento substancial na transferência de recursos financeiros para o SUS.

As transferências do SUS, fundo a fundo, da União aos municípios, estados e DF, em bilhões de reais, considerando os blocos de financiamento (atenção básica, atenção de média e alta complexidade ambulatorial e hospitalar, vigilância em saúde, assistência farmacêutica, gestão do SUS e investimentos na rede de serviços de saúde), passaram de 25,9 bilhões de reais, em 2002, para 66,79 bilhões de reais, em 2014 - um aumento de 162,5\%.

A média per capita das despesas em saúde das três esferas de governo, em reais, atualizados pelo Índice Nacional de Preços ao Consumidor Amplo (IPCA) de janeiro de 2015, por transferências de fundo a fundo e de pagamentos diretos, em 2002, passou de $\mathrm{R} \$ 159$ para $\mathrm{R} \$ 357,1$, em 2014, representando um aumento de $122,6 \%$.

A estabilidade na transferência e no aumento de recursos financeiros deve-se, incialmente, à Emenda Constitucional n ${ }^{\circ} 29$, de 13 de setembro de 2000, ao vincular recursos da União, dos estados, do Distrito Federal e dos municípios para serem obrigatoriamente gastos em ações e serviços públicos de saúde (MELAMED; PIOLA, 2011). Esse instrumento legal foi o meio usado para cobrir o fim da 
Contribuição Provisória sobre Movimentação Financeira (CPMF), em 2007, e manter o nível de investimento crescente.

Destaca-se que a Lei complementar $\mathrm{n}^{\mathrm{o}}$ 141, de 13 de janeiro de 2012, estabelece a porcentagem mínima da arrecadação dos impostos a ser aplicada em ações e serviços públicos de saúde pelos estados, Distrito Federal e municípios.

\section{Indicadores de acesso aos serviços de saúde}

Os indicadores de acesso aos serviços de saúde apresentam a evolução das internações de média complexidade sensíveis e não sensíveis à atenção básica e as internações de alta complexidade, de 2008 a 2014.

Observa-se um aumento contínuo, de 2008 a 2014, da razão entre população e internações de alta complexidade, que passou de 2,29 para 3,2, o que leva a inferir que houve investimento e um maior acesso a esse tipo de serviço, que não é sensível às ações de atenção básica e ambulatorial e que apresenta um custo mais elevado.

Quanto à razão entre a população e as internações de média complexidade, excluídas as sensíveis à atenção básica, no período estudado, verifica-se que houve pequeno decréscimo de 0,2, passando de 1,24, em 2008, para 1,22, em 2014, com flutuações no período. Esse resultado pode indicar uma discreta melhoria na organização de serviços ambulatoriais e qualificação dos recursos físicos e humanos existentes no SUS.

Com relação à razão de internações de média complexidade sensíveis à atenção básica, no período, houve decréscimo de 0,23 , sendo que, a partir de 2010, o decréscimo foi constante, passando de 1,19, no referido ano, para 0,94, em 2014. Pode-se inferir que essa diminuição da razão se deve a uma maior hierarquização do SUS com maior entrada no sistema de saúde pela atenção básica.

Verifica-se que houve um aumento na proporção de pessoas que consultaram médicos entre os anos de 2003 e 2013, passando de $62,8 \%$ para $71,2 \%$, e que houve redução na proporção da população que refere internação hospitalar nos anos de 2003 a 2013, passando de $7 \%$ para $6 \%$.

Assim, infere-se que a maior oferta de consultas médicas para a população brasileira acarretou diminuição das internações.

\section{Indicadores de recursos humanos do SUS}

A tabela 1 apresenta a evolução de dados relacionados à atenção básica: média mensal de EqSF e carga horária dispensada pelos profissionais do SUS à população, de 2008 a 2014.

Tabela 1. Média mensal de Equipes de Saúde da Família (EqSF) e carga horária dispensada pelos profissionais do SUS à população, de 2008 a 2014

\begin{tabular}{|c|c|c|c|c|c|c|c|}
\hline Indicadores & 2008 & 2009 & 2010 & 2011 & 2012 & 2013 & 2014 \\
\hline Média mensal de EqSF & 29.610 & 30.914 & 32.172 & 32.934 & 34.026 & 35.638 & 41.613 \\
\hline \multicolumn{8}{|l|}{ Razão entre horas semanais: } \\
\hline Médicos & 97 & 99,8 & 105,6 & 112,1 & 116,2 & 114,8 & 131,3 \\
\hline Enfermeiros & 86,9 & 91,9 & 96,6 & 99 & 103 & 103,6 & 111,1 \\
\hline $\begin{array}{l}\text { Outros profissionais de nível } \\
\text { superior }\end{array}$ & 31,5 & 38,1 & 44,4 & 47,7 & 52 & 55,7 & 65,3 \\
\hline
\end{tabular}


No período de 2008 a 2014, houve um aumento de 40,5\% no número de EqSF, passando de 29.610 para 41.613 equipes. Percebese que, durante o período estudado, houve um aumento progressivo no número de EqSF, com um crescimento mais acentuado em 2014, devido ao Programa Mais Médicos, que passou a contar com 18.240 médicos.

Com relação à razão de horas semanais de médicos e população residente na atenção básica, observou-se o aumento de 35,4\%, entre 2008 e 2014 , em que a média passa de 97 para 131,3 .

Destaca-se o aumento mais acentuado no período entre 2013 e 2014, em que a razão de horas semanais passou de 114,8 para 131,3 horas. Esse aumento deve-se à implantação do Programa Mais Médicos, que, em dezembro de 2014, contou com o ingresso de 13.825 médicos para atendimento na atenção básica, distribuídos em 3.714 municípios em todos os estados do País.

No tocante à razão de horas semanais entre enfermeiros na atenção básica e população residente, verifica-se que houve aumento decorrente, principalmente, do aumento da carga horária semanal dos enfermeiros da atenção básica vinculados ao SUS no País. Essa razão passou de 86,9, em 2008, para 111,1, em 2014, com um aumento de $35,4 \%$.

E a razão de horas semanais de outros profissionais de nível superior mais que dobrou entre 2008 e 2014 . Apresentou um aumento de $107,3 \%$, passando de 31,5 para 65,3 horas. Verifica-se o maior incremento em 2014, o que indica maior incentivo na implantação de equipes multiprofissionais na atenção básica.

\section{Considerações finais}

Avaliar o desempenho de um sistema de saúde é uma tarefa bastante complexa devido aos inúmeros determinantes inter-relacionados que influenciam a condição de saúde de uma população. Todo sistema de saúde está intrinsicamente organizado e inserido em um contexto político-social que o determina e a quem ele diretamente influencia. É essa interação entre determinantes gerais e respostas específicas que tem sido motivo de preocupação de diversos autores. Castellanos (1997) é um desses estudiosos. Ele entende que a avaliação de um sistema de saúde pressupõe uma ação para modificar a situação de saúde da população.

Tendo em consideração que a organização de um sistema de saúde é simultaneamente determinada pela situação de saúde da população da área de abrangência e pela resposta organizada a essa situação, é fundamental, para a análise do desempenho, envolver e compreender os determinantes das condições de vida e saúde da população estudada.

Outra questão que deve ser considerada, quando da avaliação do desempenho, é se os objetivos do sistema de saúde estão claramente definidos, pois, para medir o alcance, é necessário que os objetivos estejam explícitos e, de preferência, pactuados entre os atores envolvidos.

Santos (2015), em seu estudo, traz uma definição abrangente de sistema de saúde e mostra a complexidade e amplitude do tema, que é avaliar um sistema de saúde, quando escreve

a definição de um sistema de saúde tem um conjunto das intervenções que têm como meta problemas específicos, sociais ou de saúde; com uma série de intervenções: de serviços preventivos aos serviços paliativos, passando pelos serviços de diagnósticos e curativos. Compreendem as grandes funções da saúde pública: vigilância, proteção e promoção da saúde, prevenção das doenças, avaliação do sistema dos serviços de saúde, desenvolvimento das competências em saúde pública. Entendo que os sistemas de saúde não têm responsabilidades diretas ou governabilidade sobre o conjunto das condições sociais, econômicas, culturais e demográficas que afetam a capacidade das pessoas de viver bem e por muito tempo. (SANTOS, 2015, P. 34-35).

Podemos, ainda, acrescentar a essa definição 
questões importantes a um sistema de saúde não contempladas, como a reabilitação física e profissional, o controle social e a integralidade com as ações relacionadas à seguridade social.

A criação de um índice, que correlacione indicadores, é ainda uma questão polêmica no campo da saúde pública. As iniciativas da Organização Mundial da Saúde (OMS), por exemplo, de estabelecer um índice mundial e do Ministério da Saúde, com o IDSUS, geraram muitos questionamentos e reações. A matriz de avaliação do Proadess, por outro lado, é uma importante iniciativa no sentido de avaliar um sistema de saúde, pois faz uma descrição extensa dos elementos constitutivos de um sistema, suas dimensões e subdimensões, e dos indicadores que os expressam, porém, não comtempla questões importantes do setor saúde, como a vigilância epidemiológica e sanitária, a promoção da saúde, entre outros temas da saúde coletiva. Outro aspecto a ser destacado na matriz do Proadess é que ela tangencia a necessidade de uma definição clara e abrangente dos objetivos para avaliar desempenho. Trata-se, portanto, de um modelo de análise de desempenho que necessita de aprimoramentos e de outros insumos para completar a análise do desempenho.

Analisar o desempenho de um sistema como o SUS, que não tem comando único, organizado em realidades desiguais, com demandas diferenciadas, culturas diversas e, principalmente, por envolver condicionantes e estruturas de governos municipais, estaduais e do governo federal, é realmente desafiador.

Todavia, a definição de Santos (2015) norteou a realização de uma análise crítica sobre ' $\mathrm{O}$ SUS em vista'. Na perspectiva de enfrentar esse desafio é que foi elaborado 'O SUS em vista', que parte de uma definição tácita sobre sistema de saúde e seus objetivos e que escolhe indicadores, relações e agrupamentos para avaliar o desempenho do SUS sem explicitar os critérios utilizados. Baseado nisso, foi feita a escolha e o agrupamento dos indicadores em: socioeconômicos; de saúde; de financiamento; e de serviço de saúde do SUS não são explicitados.
Levando em consideração as limitações acima discutidas, o presente artigo se constitui em uma iniciativa, ainda que parcial, de fornecer e correlacionar dados e informações para subsidiar estudos mais detalhados e localizados sobre o desempenho do SUS.

O artigo mostra que, no período de 2002 a 2014, houve uma sensível melhoria em um conjunto de indicadores tanto de natureza socioeconômica quanto de condições de saúde. A melhoria dos indicadores de condições de saúde está provavelmente relacionada: ao incremento de suporte financeiro e de recursos humanos; ao aumento do acesso às consultas médicas básicas e aos serviços de alta complexidade; e à maior disponibilização de horas de profissionais de saúde para a população residente, conforme apresentado.

Destaca-se a inovação do indicador, que demonstrou um aumento na razão de horas semanais de médicos, enfermeiros e outros profissionais de saúde de nível superior na atenção básica, indicando mais contato e maior vínculo entre profissional de saúde e população residente. Anteriormente, esse dado era apresentado apenas no montante de recursos humanos através do número de profissionais.

O acompanhamento regular, em curtos espaços, de indicadores gerais e específicos, como o indicador de horas entre profissional e usuário, permite ajustar rotas e escolhas e corrigir equívocos (JANNUZZI, 2012). Nesse sentido, é importante valorizar estudos transversais sobre os indicadores que avaliam o desempenho do SUS em suas dimensões constitutivas.

Entretanto, faz-se necessário ampliar o debate e insistir no caminho de construir um índice geral de desempenho do SUS que entenda e contemple a complexidade de ações, com a necessária participação dos atores envolvidos. O índice do SUS pode ser um poderoso instrumento de gestão para as três esferas de governo, pois permite a síntese de dimensões distintas, assim como permite o acompanhamento sistemático da implantação de ações de saúde ao longo do tempo, apoiando a gestão na tomada de decisões. 


\section{Referências}

BRASIL. Ministério do Planejamento, Orçamento e Gestão. Secretaria de Planejamento e Investimentos Estratégicos. Plano plurianual 2012-2015: projeto de lei. Brasília, DF: Ministério do Planejamento, 2011. Disponível em: <http://www.planejamento.gov.br/ secretarias/upload/arquivo/spi-1/ppa-1/2012/mensagem_presidencial_ppa-2.pdf>. Acesso: 15 abr. 2016.

Ministério da Saúde. Portaria no 399, de 22 de fevereiro de 2006. Divulga o Pacto pela Saúde 2006 Consolidação do SUS e aprova as Diretrizes Operacionais do Referido Pacto. Brasília, DF, 22 fev. 2006. Disponível em: <http://bvsms.saude.gov.br/bvs/saudelegis/ gm/2006/prt0399_22_02_2006.html>. Acesso em: 14 mar. 2016.

Ministério da Saúde. Secretaria de Atenção à Saúde. Departamento de Atenção Básica. Pacto de Indicadores da Atenção Básica: instrumento de negociação qualificador do processo de gestão do SUS.

Revista Brasileira de Saúde Materno Infantil, Recife, v. 3, n. 2, p. 221-224, abr./jun. 2003. Disponível em: <http:// www.scielo.br/pdf/rbsmi/v3n2/al3v03n2.pdf > . Acesso em: 15 mar. 2016.

Ministério da Saúde. Secretaria Executiva. Departamento de Monitoramento e Avaliação do SUS. IDSUS: Índice de Desempenho do Sistema Único de Saúde. 2012. Disponível em: <http://idsus.saude.gov.br/ documentos.html>. Acesso: 15 mar. 2016

Ministério da Saúde. Secretaria Executiva. Departamento de Monitoramento e Avaliação do SUS. O SUS em vista: indicadores socioeconômicos, de saúde, de financiamento e de serviços de saúde. Brasília, DF: Ministério da Saúde, 2016. Disponível em: <https://drive.google.com/ file/d/OB3VoXrhG0ORWWW5ydıNTY3BfOE0/ view? $\mathrm{usp}=$ sharing $>$. Acesso em: 25 out. 2016.

Ministério da Saúde. Secretaria de Gestão Estratégica e Participativa. Departamento de Articulação Interfederativa. Caderno de Diretrizes, Objetivos, Metas e Indicadores: 2013-2015. 3 ed. Brasília, DF: Ministério da Saúde, 2015. (Série Articulação
Interfederativa; v. 1). Disponível: <http://189.28.128.100/ sispacto/CadernoDiretrizes2013_2015.pdf >. Acesso em: 16 fev. 2017.

CAMPELlO, T. (Org.). Programa Bolsa Família: uma década de inclusão e cidadania. Brasília, DF: Ipea, 2013.

CASTELLANOS, P. L. Epidemiologia, Saúde Pública, Situação de Saúde e Condições de Vida. Considerações conceituais. In: BARATA, R. C. B. (Org.). Condições de vida e saúde. Rio de Janeiro: Abrasco, 1997.

DUARTE, E. C. et al . Expectativa de vida ao nascer e mortalidade no Brasil em 1999: análise exploratória dos diferenciais regionais. Revista Panamericana de Salud Publica, Washington, v. 12, n. 6, p. 436-444, dez. 2002.

GESSER, H.C. Expectativa de vida no Brasil: a geração de modelos explicativos por regressão linear múltipla. 2005. 137 f. Tese (Doutorado em Engenharia de Produção) - Programa de Pós-graduação em Engenharia de Produção, Universidade Federal de Santa Catarina, 2005.

\section{INSTITUTO DE PESQUISA ECONÔMICA}

APLIACDA (IPEA). Duas décadas de desigualdade e pobreza no Brasil medidas pela PNAD/IBGE. Brasília, DF: Ipea, 2012. (Comunicados do Ipea; 155). Disponível em: <http://www.ipea.gov.br/agencia/images/stories/ PDFs/comunicado/120925_comunicadodoipeal55_ v5.pdf.>. Acesso: 1 abr. 2016.

Evolução do analfabetismo e do analfabetismo funcional no Brasil: Período 2004-2009. Brasília, DF: Ipea, 2010. (Comunicados do Ipea; 70). Disponível em: <http://www.ipea.gov.br/portal/images/stories/PDFs/ comunicado/101209_comunicadoipea70.pdf $>$. Acesso: 1 abr. 2016

Objetivos de desenvolvimento do milênio: relatório nacional de acompanhamento. Brasília, DF: Ipea, 2014

JANUZZI, P. Indicadores sociais no Brasil. Campinas: Alínea, 2012. 
MEDICI, A. O Índice de Desempenho do SUS (IDSUS). Monitor de Saúde, [S.1.], ano 7, n. 35, mar.

2012. Disponível em: <http://monitordesaude.blogspot. com/2012/03/o-indice-de-desempenho-do-sus-idsus. html>. Acesso: 14 mar. 2016.

MELAMED, C.; PIOLA, S. F. (Org.). Políticas públicas e financiamento federal do Sistema Único de Saúde. Brasília, DF: Ipea, 2011.

FUNDAÇÃO OSWALDO CRUZ (FIOCRUZ). Projeto de Desenvolvimento de metodologia de avaliação do desempenho do sistema de saúde brasileiro (PROADESS): relatório final. Rio de Janeiro: Fiocruz, 2003. Disponível em: <http://www.proadess.icict.fiocruz.br/ relatoriofinal.pdf>. Acesso: 1 mar. 2016.

\section{Avaliação de Desempenho do Sistema de Saúde}

Brasileiro: indicadores para monitoramento: relatório final. Rio de Janeiro: Fiocruz, 2011. Disponível em: $<$ http://www.proadess.icict.fiocruz.br/SGDP-

RELATORIOFINAL24-5-2011.pdf>. Acesso: 9 mar. 2016.

REDE INTERAGENCIAL DE INFORMAÇÃO PARA A SAÚDE (RIPSA). Indicadores básicos para a saúde no Brasil: conceitos e aplicações. 2. ed. Brasília, DF: Organização Pan-Americana da Saúde, 2008.

SANTOS, L. C. Crítica a modelos de avaliação de desempenho de sistemas de saúde. 2015. 146 f. Tese (Doutorado em Saúde Pública) - Faculdade de Saúde Pública, Universidade de São Paulo, São Paulo, 2015.

Recebido para publicação em abril de 2016

Versão final em novembro de 2016

Conflito de interesses: inexistente

Suporte financeiro: não houve 